\title{
An Impacted Foreign Body (A Betel Nut) in Umbilicus with Umbilical Hernia
}

\author{
Dr. Vikrant Patel ${ }^{1}$, Dr. Iliyas Juneja ${ }^{2}$, Dr Bhavesh V. Vaishnani ${ }^{3}$, Dr. Jatin Bhatt ${ }^{4}$ \\ ${ }^{1}$ Resident Doctor, PDU Medical College, Rajkot, Gujarat, India \\ ${ }^{2}$ Assistant Professor, PDU Medical College, Rajkot, Gujarat, India \\ ${ }^{3}$ Associate professor, PDU medical college, Rajkot, Gujarat, India \\ ${ }^{4} \mathrm{HOD}$ and professor, PDU medical college, Rajkot, Gujarat, India
}

\begin{abstract}
Introduction: Polyembolokoilamania1 (inserting a foreign body into 1 body orifice or more) is a psychiatric disorder. Here patient had the habit of reducing the umbilical hernia with a betel nut.On one such attempt he failed to do so and the betel nut got stuck in the umbilicus. Case Report: A 56 year old male Hindu patient of low socio-economic class presented with the chief complaint of painful swelling over the umbilicus with serous discharge since 4 days with foreign body in situ since 8 days. Patient used to reduce umbilical hernia with the help of a betel nut. Patient had inserted it before 8 days but failed to remove it and pain and swelling increased. Patient had no associated complaints of vomiting, constipation, distension, fever. Management: A small transverse incision was made and betel nut was removed under G.A. with Omphalectomy done and Umbilical hernia defect repaired and meshplasty done with prolene mesh. Post operative period was uneventful. Discussion: Case reports of insertion /impaction of foreign body are associated with self injurious behaviour characteristics of bipolar disorder. However some cases have been thought secondary to Munchausen syndrome or secondary gain. In this type of psychiatric illness mostly foreign body is inserted into anus, urethra or vagina.
\end{abstract}

Keywords: betel nut; foreign body; umbilical hernia; psychiatric illness; omphalectomy; meshplasty

\section{Introduction}

Polyembolokoilamania $^{1}$ (inserting a foreign body into 1 body orifice or more) is a psychiatric disorder. An umbilical hernia $^{2}$ is a sac formed from the inner lining of your abdominal cavity that pushes through a hole in the abdominal wall at the belly button. Here patient had the habit of reducing the umbilical hernia with a betel nut .On one such attempt he failed to do so and the betel nut got stuck in the umbilicus.

\section{Case Report}

A 56 year old male Hindu patient of low socio-economic class presented with the chief complaint of painful swelling over the umbilicus with serous discharge since 4 days with foreign body in situ since 8 days.

Patient has umbilical swelling since 6 months. Initially the size of the swelling was similar to that of a grape. Then the size increased as the time progressed. The swelling reduced by itself on lying down in supine position. The swelling would again become prominent on standing or on exertion. The size of the swelling increased to that of a lemon in 2 months time. Patient used to keep the umbilical hernia in reduced state with help of a betel nut. Then after some time the patient used to remove it. But every time he removed it, umbilical hernia would appear again on exertion. Patient had made 3 such attempts in the past .But 8 days back when the patient inserted the betel nut to reduce the hernia, he failed to remove it and as a result he developed severe pain around the umbilicus. Gradually the intensity and severity of pain increased. It was associated with umbilical discharge and redness around umbilical skin. Patient came to civil hospital Rajkot with the betel nut in-situ which was visible as well as palpable. So the patient was admitted and planned for an emergency operative procedure.
Patient had no associated complaints of vomiting, constipation, distension, fever.

On examination: redness, edema, tenderness around umbilical skin with foreign body in situ. Foreign body was palpable during digital examination at local site.

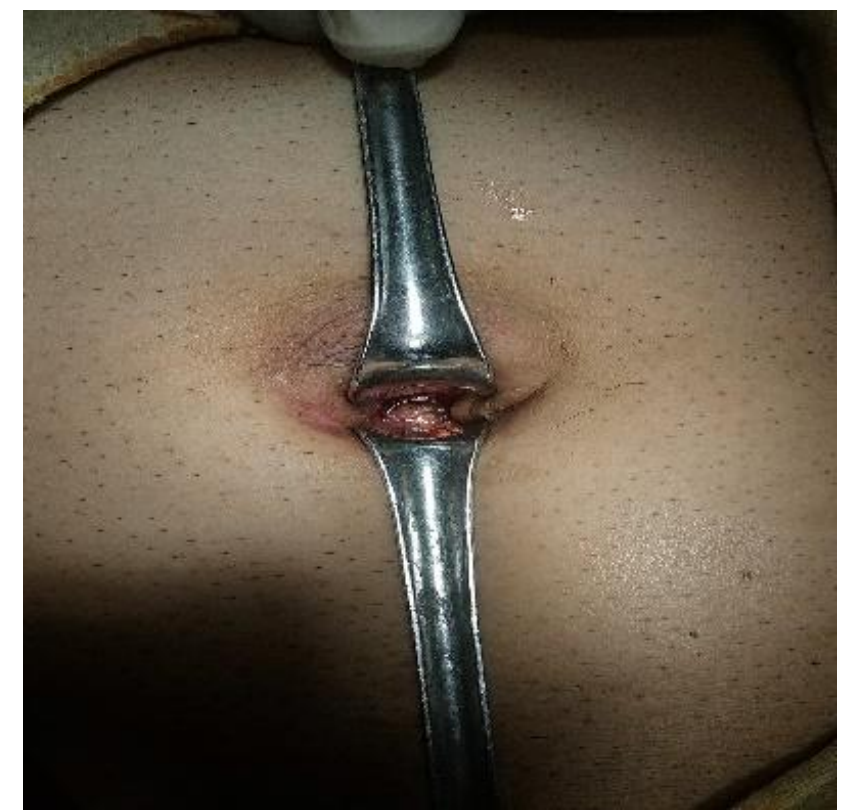

On retraction of the skin around the umbilicus foreign body was visible.

Our patient underwent $\mathrm{X}$-Ray chest and $\mathrm{X}$ ray abdomen standing which wasnot of any significance. On ultrasonography no any significant pathology was noted.

Patient was a K/C/O a psychiatric illness for which he had undergone electroconvulsive therapy 3 times before 25 yrs. Patient himself did not have any significant knowledge 


\section{International Journal of Science and Research (IJSR) \\ ISSN (Online): 2319-7064}

Index Copernicus Value (2013): 6.14 | Impact Factor (2014): 5.611

regarding the psychiatric illness. Patient is not on any psychiatric treatment at present.

\section{Management}

Patient was taken to operation table. Antiseptic precautions taken. An attempt was made to remove betel nut with help of Allis forceps an artery forceps without anaesthesia. It failed as the procedure was very painful. So general anaesthesia was given and operative procedure started. A small transverse incision was made and betel nut removed.

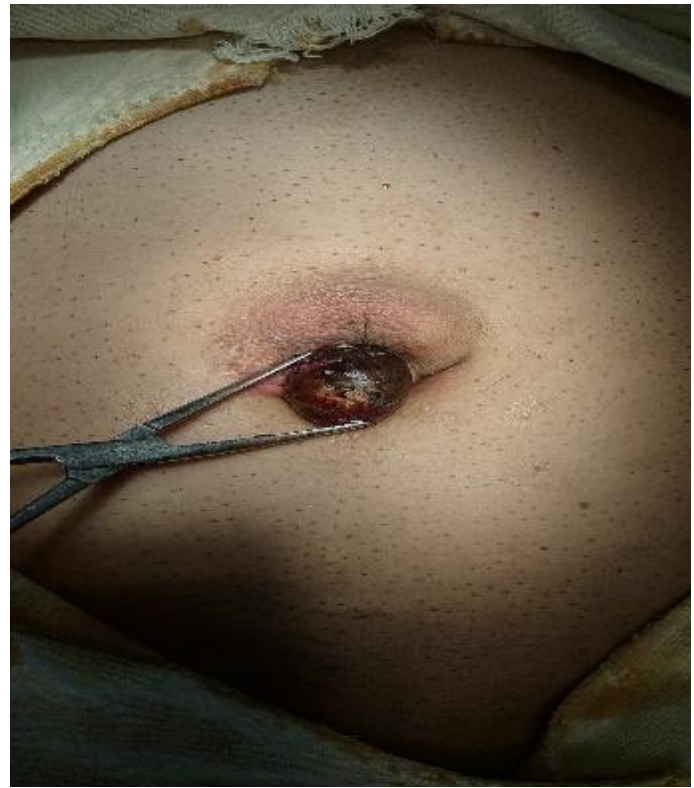

Omphalectomy done then Umbilical hernia defect repair with prolene meshplasty with mesh size $(7.5 \mathrm{cms} * 15 \mathrm{cms})$ under G.A. A negative suction drain kept in subcutaneous plane on right side to umbilicus.

Negative suction drain removed on-4th post operative day Patient discharged on $-4^{\text {th }}$ post operative day

On discharge-wound clean.

Post operative period - uneventful.

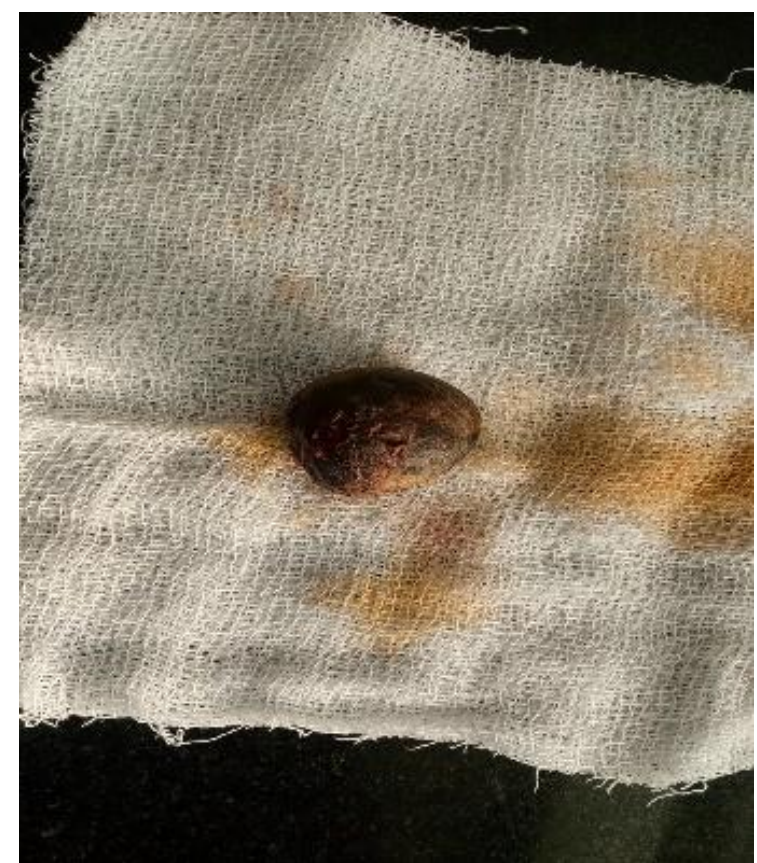

\section{Discussion}

In history, a several methods were used to reduce or to prevent hernia. Truss ${ }^{3}$ and hernia belts were used since many years. For use of these material hernias must be reducible. Recently in some rural area only these type of practice used. Truss for inguinal hernia works quite good, but truss for umbilical hernia it can worsen hernia as it will increase intra-abdominal pressure and it forces hernia out with more force than what is applied directly to hernia by belt. So ideally they are useless.

The present case is definitely foreign body induced omphalitis ${ }^{4}$. Hair ball is the most common type of foreign body seen in such cases. It's called lint-ball omphalitis ${ }^{5}$. Such patients do not maintain a proper local hygiene. In adult its alarming as can caused by many congenital or acquired conditions like patent urachus, urachal cyst, urachal sinus, patent vitteline duct, vitteline cyst or sinus. Acquired causes are like pilonidal sinus disease, infction, acute or chronic inflammation or abscess of umbilicus. Rarely endometriosis or metastatic carcinoma.

Case reports of insertion /impaction of foreign body are associated with self injurious behaviour characteristics of bipolar disorder. However some cases have been thought secondary to Munchausen syndrome ${ }^{6}$ or secondary gain. In this type of psychiatric illness mostly foreign body inserted into anus, urethra or vagina.

\section{References}

[1] http://www.ncbi.nlm.nih.gov/pmc/articles/PMC371925 $0 /$

[2] http://www.healthline.com/health/umbilical-hernia

[3] https://en.wikipedia.org/wiki/Truss_ (medicine)

[4] http://www.gpnotebook.co.uk/simplepage.cfm?ID=x20 130803150824685340

[5] http://www.ncbi.nlm.nih.gov/pmc/articles/PMC274022 9/

[6] https://en.wikipedia.org/wiki/Munchausen_syndrome 